\title{
Multiple Mandibular Tori: Three Case Reports And Review.
}

\author{
Dr. Manjusha S.Pradhan ${ }^{1}$, Dr.Swapnil N.Patil ${ }^{2}$, Dr.Varsha S.Uttarwar ${ }^{3}$, \\ Dr.Vidya A.Mokhade \\ ${ }^{1,2,3,4}$ (Department Of Conservative Dentistry, VSPM Dental College And Research Centre,Maharashtra \\ University Of Health Sciences,Nashik/University,India)
}

\begin{abstract}
Multiple Mandibular Tori are common localized overgrowths of the bone. They are non-neoplatic and are thought to be reactive or developmental in origin. These need to be accurately distinguished from other more diagnostically significant lesions. The etiology of the Mandibular tori has been investigated by different authors, but no consensus has been reached so far. We are reporting three cases of an otherwise healthy patients from the age group of 45-65 years with Mandibular Tori, which were correlated both clinically and radiographically.
\end{abstract}

Keywords: Tori, Torus Mandibularis, Totrus palatinus

\section{Introduction}

The tori meaning 'to stand out"or 'lump' in latin which is define as a bony protuberance with benign characteristic, leading to the "overworking of osteoblasts" and bone to be deposited along the line of fusion of the palate or on the hemi-mandibular bodies.[1] The tori or exostosis are formed by a dense cortical and limited amount of bone marrow, and are covered with a thin and poorly vacularised mucose which creates a hard visible mass in the oral cavity [2] .The peak incidence of Torus Palatinus(TP) and Torus Mandibularis (TM) is in the third decade of life [3].Occurence of TP is more in women than men and the occurrence of TM is higher in men than in women $[2,3,4]$. They begin to develop in early adulthood and may enlarge very slowly over the years. TP can be flat, nodular,lobolar or spindle shaped .[1,5] TM are usually nodular, unilateral or bilateral and single or multiple while exostosis are multiple bony nodules [2]There are three types of Tori which were named on the basis of their location in the mouth. These entities are very site specific

1. Torus Palatinus is found only in the middle of the hard palate. $[5,6]$

2. Torus Mandibularis is found only in the lingual surface of the mandible,near the bicuspid teeth,they are usually bilateral. $[2,5,6]$

3---Buccal exostosis also called as Bony Exostosis found only on facial or outer aspect of the upper jaw and less commonly the lower jaw.[7]

Although there is no clear criteria for classification by size ,classification into two groups of 1) $2 \mathrm{~mm}$, $2-4 \mathrm{~mm}$, and above $4 \mathrm{~mm}$,or 2) $3 \mathrm{~mm}, 3-6 \mathrm{~mm}$, and above $6 \mathrm{~mm} .2 \mathrm{~mm}$ and $3 \mathrm{~mm}$ have been found to be common sizes. [2] Usually tori are $1.5 \mathrm{~mm}-4 \mathrm{cms}$ in diameter and can be single growth or grouped together in a cluster i.e. nodular .Buccal exostosis occur less frequently than Tori. Buccal Exostosis and tori are benign lesions and do not passess malignant potential[1,2].

The exact cause of appearance is not clear $[1,2,6,8,9]$. The most accepted theory of TM today is of genetic origin in $29.5 \%$ cases and $70 \%$ cases are related to increased masticatory forces to the teeth i.e. occlusal stress.[2,8]Another cause is superficial injuries or its occurrence as a functional response in individuals with well developed chewing muscles.or in patients with abraded teeth due to occlusion Reichart et.al. [10],Andres S. Garcia et al[1] and Basha S. et al [9] found a significant co-relation between the incidence of Torus and the presence of abraded teeth. Cliford et.al. [11] and Kerdpon et.al.[12] found a large relationship between the TM and parafunctional habits, which they did not find with the TP .Thus Sirirumgrojying et al.[13] determined that TM can be used to indicate the risk of appearance of tempero mandibular disorders. It can also be due to eating habits, states of vitamin deficiency or dietary supplements rich in calcium. It could be also associated with consumption of fish, because fish contains omega 3 unsaturated fatty acids and vitamin $\mathrm{D}$, which encourages bone growth. It may be because of prolonged use of phenytoin which leads to an increase in size of torus as it induces an increase in calcium homeostasis functioning as an osteogenic agent. [1] The majority of these Tori are asymptomatic , and remain undisturbed over the patient's lifetime . $[1,14]$ and observed during clinical examination at the dental clinic. Sometimes patients may present phonetory disturbances, limitation of masticatory mechanics, ulcerations of the overlying mucosa ,food deposits, periodontitis, prosthetic instability and some patients may have cancer phobia.$[1,2,6,15]$ In addition to that, TM may cause sleep apnea or difficulty of intubation under G. A.[2] 
Tori can frequently be confused with other conditios -

1 - oral cancer---cancerous tumors are soft to touch, while Tori feel hard and bony

2 -impacted / unerupted teeth

3 - tooth abscess

4-mouth ulcers /canker sores

5 -salivary gland swelling

Shubha rajan dutta et al [15] states that TM are not associated with any pathological condition \& the condition remains asymptomatic, slowly enlarging recurrent lesions are occasionally seen, but there is no malignant transformation potential. Treatment usually consist of surgical reduction of the lesion or chiselling of bone to the level of surrounding bone by the dentist experienced in removing Tori.

\section{Case report}

A 62 yrs male engineer reported to the clinic with a chief complaint of pain in lower right back region since last 2 months. The patient was diabetic and hypertensive since last 10 years, and on medication for the same. On clinical examination patient had badly carious $46 \& 47$ with generalized attrition. Soft tissue examination revealed a bony growth extending bilaterally from 44 to 46, which was hard on palpation, suggestive of bony extention/tori. Occlusal radiograph showed radiopaque mass extending bilaterally from 44 to 46 , and 34 to 36 without lymphadenopathy. Patient was totally asymptomatic and unaware of these extensive bony growths.(fig.1,2)

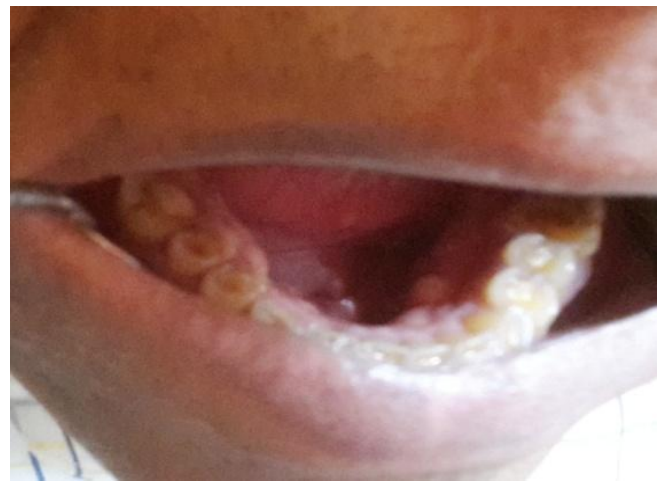

Fig.1- Mandibular tori bilateral

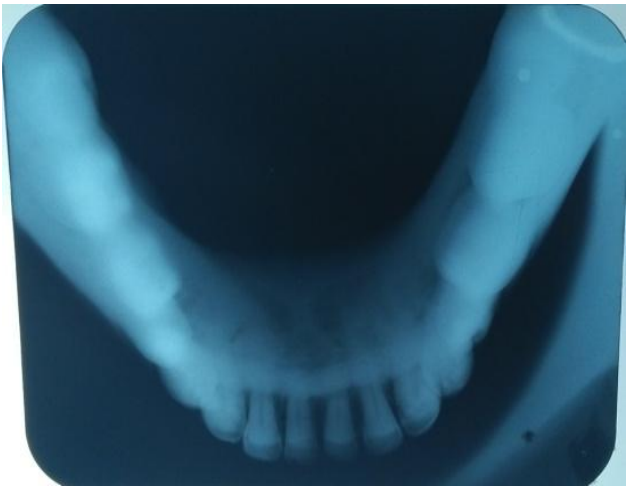

Fig.2. Occlusal Radiograph showing tori

\section{Case report}

A 55 yrs male pharmacist reported to the clinic with chief complaint of pain in upper right back region since 15 days. At the time of visit patient was in good general health with no history of Diabetes Mellitus or Hypertension. On clinical examination, patient had badly carious 37, and with 27 RCT was done with full metal crown. Soft tissue examination revealed bony growth extending bilaterally from lower lateral incisor to first molar which was hard on palpation and non tender with normal mucosa covering growth,suggestive of Tori. Occlusal radiograph showed radiopaque mass extending bilaterally from 32 to 36 and 42 to 46 . Patient was totally asymptomatic and was unaware of bony nodular growth in the oral cavity.(fig. 3,4$)$

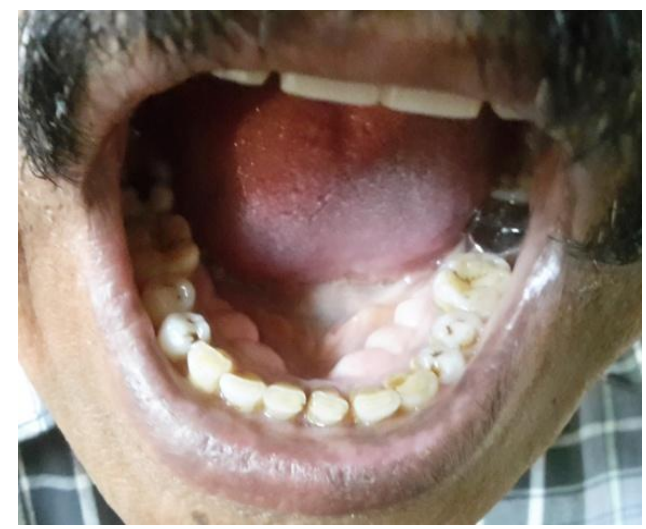

Fig.3. Bilateral Mandibular tori

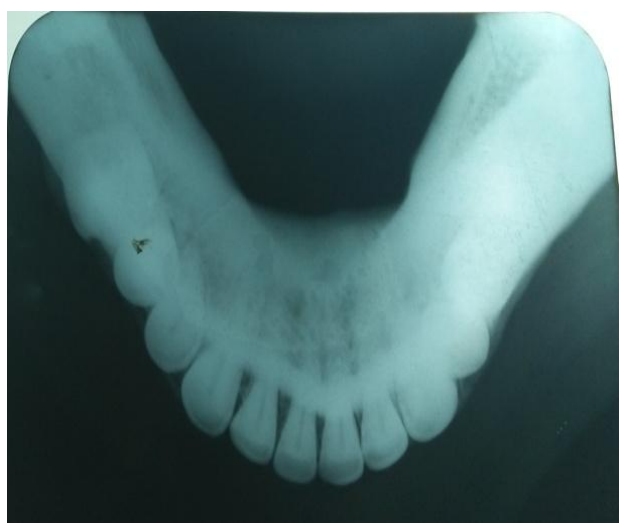

Fig.2. Occlusal Radiograph 
Case report 3: A female medical professional patient aged 53, came to the clinic for routine check up and oral prophylaxis. Patient was in good general health with recently diagnosed Diabetes Mellitus which was controlled with diet and exercises. On clinical examination her oral hygiene was good .Upper deciduous canines were over retained and carious with rest of sound intact dentition. On examination, multiple bony nodules were evident in the lingual region below tongue extending from lower lateral incisor to second premolar bilaterally, which was hard on palpation, with normal covering mucosa, suggestive of Tori. Patient was totally asymptomatic without lymphadenopathy, and was well aware of the presence of the bony growth in the oral cavity. As the growth was asymptomatic and she was well aware of the condition, she was not bothered about it and refused to take radiograph. (fig.5)

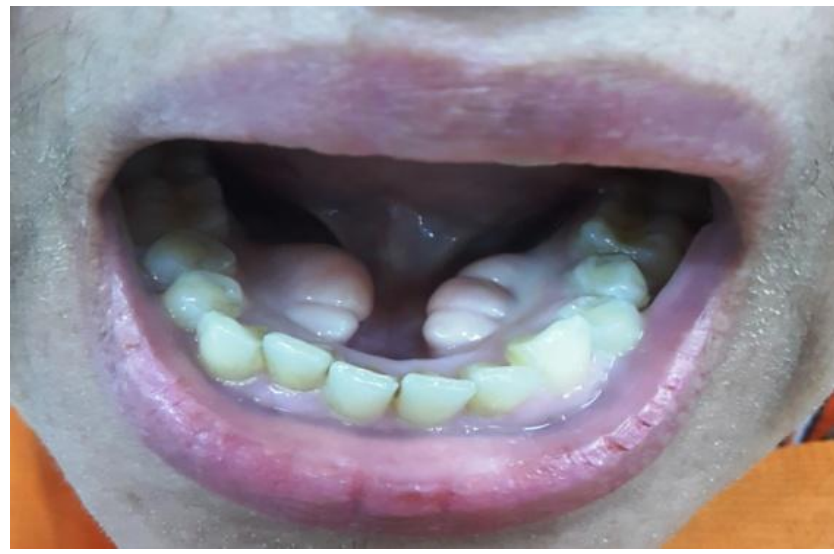

Fig 5.Large Bilateral mandibular tori

\section{Discussion}

According to Salman Basha et al [9] TM is recognized as a bony ridge or series of bony nodules or lumps appearing on the lingual surface of the alveolar margin of the mandible, generally in the premolar region. In our cases we also found the tori in premolar region. According to Subha rajan dutta et al [15], Tempero Mandibularis ,commonly called Mandibular Tori are asymptomatic benign bony masses and exhibit a relatively high prevalence and it has been reported in the literature for over $180 \mathrm{yrs}$. In our cases all the tori are mandibular tori suggestive of high prevalence.

In the study by Youna Choi et al[16] ,they stated that the prevalence of these anomalies increases with age ; and found that Tori develop in the $2^{\text {nd }}$ or $3^{\text {rd }}$ decade of life ,initially growing rapidly .the growth rate is then thought to decrease with age ,but never stops. In our cases the patients were from age group 45-65 years suggestive of growth of tori that never stops. Exact cause or etiology of Tori is not known but Andres S.Garcia et al [1] found that ,the most accepted cause of TM is of genetic origin, or due to Occlusal stress.[17] In our cases one patient has generalized attrition which seconded that the occlusal stress as one of the etiological factor for TM.Studies done by Shubha Rajan Dutta et al [15] found that , TM arises on the lingual side of the jaw, in the region of the Premolars or bicuspids generally above the location of the mylohyoid muscles attachment to the mandible and may extend to molar region. Similar findings were seen in our cases also, the tori were extended from lateral incisors to molars According to Salman Basha et al [9] and Shubha Rajan Dutta et al [15] TM generally manifested bilaterally, forming hard, rounded swelling. Similarly, in our cases they are also found bilaterally. Shubha Rajan Dutta et al [15] also found that occurrence of TM slightly more in men than in women and TP are more common in women than men.[3,4,15] In our cases out of 3 patients, 2 were male patients . According to Salman Basha et al [9] the torus may be bosselated or multilobulated. Manasi bansal et al [18] and Youna Choi et al[16] have shown that Tori consists of prominent hard tissue with a thin mucosa and it has a normal appearance .But traumatic ulcers may easily develop at the site if it is exposed to trauma and then it may become whitish and ulcerative. In all the 3 cases mentioned, there was no history of trauma or ulceration and mucosa was absolutely normal with no lymphdinopathy.

\section{Treatment}

Generally surgical resection is not required for TM as long as the condition remains asymptomatic..And unless it becomes so large that 1 - it interferes with function or denture placement.

2---suffers from recurrant traumatic surface ulcerations because of sharp food ,such as potato chips or fish bones.

3---contributing to a periodontal condition.

DOI: 10.9790/0853-1606072629 $\quad$ www.iosrjournals.org $28 \mid$ Page


4---large mandibular tori can prevent complete seating of impression trays and dentures If needed ,they are surgically removed [15]

Many researchers /clinicians suggested the potential use of Tori as autogenous bone graft.in the treatment of intrabony defects because it consists of compact bone without marrow spaces. Use of mandibular tori for bone graft tissue material in periodontology and implant surgery have also been reported. $[1,15,16,19]$ Shubha Rajan Dutta et al[15] have stated that surgical resection is not required for TM, as long as the condition remains asymptomatic.However ,it is indicated when subjective symptoms, such as discomfort, pain,articulation disorder,or problems in the insertion of dentures are present.[20,21]. In the cases presented here, even though the patients were well educated ,two male patients were not aware of the TM in the oral cavity.. Third patient, being a medical professional and because of the large size of the bilateral Tori was aware of the bony growth or condition. she was asymptomatic and have knowledge about the non-malignant condition. All the three patients did not need any treatment because they were asymptomatic. But as dental professional mandibular tori should not be ignored and should be carefully diagnosed from other conditions and should be kept under observation for any pathological changes.

\section{VI. conclusion}

Torus mandibularis is asymptomatic and does not have malignant transformation potential and so does not usually require any surgical Treatment, but only re assurance. In some situations these tori may need to be surgically removed when they are causing interference in the fabrication of prosthesis or function .All three patients after counselling had a clear concept of the condition and did not need any treatment at the time of visit. But it should not be ignored and should be carefully differentiated or diagnosed from other conditions. They were advised to have follow up for the same.

[1]. Andres S.Garcia-Garcia et al. Current status of the torus palatines and torus mandibularis.Med Oral Patol oral Cir Bucal.2010;1:15(2):e353-60

[2]. Kuk Han lee,Jong Hun Lee,Ho Jung Lee.Concurrence of torus mandibularis with multiple buccal exostoses.2013;40(4):466-468.

[3]. Jainkittivong A, Apinhasmit W,Swasdison S.Prevalence and clinical charecteristics of Oral tori in 1520 Chulalongkorn University dental school patients.Surg Radiol Anat.2007;29(2):125-31.

[4]. Shah DS,Sanghavi SJ, Chawda JD,Shah RM.Prevalence of torus palatines and torus mandibularis in 1000 patients.Indian J Dent Res.1992;3(4):107-10.

[5]. Oral \& Maxillofacial Pathology;Developmental defects of the Oral and Maxillofacial Region by Naville,Damm Allen Bouquot :Restricted South Asia edition Third Edition reprint 2009 Chapter 1,Page 672

[6]. [6] Petersons Principle of Oral and Maxillofacial Surgery ; Benign non ododntogenic lesions of the jaw by Michael Miloro; CBS Publishers \& Distributors, Third edition Volume one Chapter 30 .Page 671-73

[7]. Chandna S, Sachdeva S, Kochar D,Kapil H.Surgical Management of the bilateral Maxillary buccal exostosis. J Indian Soc Periodontol.2015;19(3):352-5

[8]. Medsinge SV et al. Buccal exostosis :a rare entity.J Int Oral Health.2015;7(5):62-4

[9]. Basha S, Dutt SC.Buccal sided mandibular angle exostosis-a rare case report.Contemp Clin Dent.2011;2(3):237-9.

[10]. Reichart PA, Neuhaus F,Sookasem M.Prevalence of torus palatinusand turus mandibularis in Germans and Thai.Community Dent Oral epidemiol.1988;16:61-4

[11]. Clifford T,Lamey PJ,Fartash L.Mandibular tori,migraine and temperomandibular disorders.Br DentJ.1996;180:382-4

[12]. Kerdpon D,Sirirungrojying S.A clinical study of oral tori in southern Thailand:prevalence and the relation to parafunctional activity.Eur J Oral Sci.1999;107:9-13.

[13]. Sirirungrojying S, Kerdpon D.Relationship between oral tori and temperomandibular disorders.Int Dent J.1999;49:101-4

[14]. Pynn BR,Kurys-Kos NS,Walker DA,Mayhall JT.Tori mandibularis:a case report and review of the literature.J Can Dent Assoc.1995;61(12):1057-8

[15]. Shubha ranjan dutta,Don Verghese,Amar Bhuibhar,Ronak Desai.Mandibular Exostosis.Dental impact.2013;5(1):28-33

[16]. Youna Choi et al.Prevalence and anatominc topography of mandibular tori:computed tomographic analysis.J Oral Maxillofac surg.2012;70:1286-91

[17]. Arthur Rodriguez Gonzalez Cortes et al;Mandibular tori are associated with mechanical strss and mandibular shape;Journal of Oral and Maxillofacial Surgery ;2014;72(11):2115-25

[18]. Mansi Bansal,Sanjay Rastogi,Anamika Sharma.Multiple Mandibular Exostosis: A Rare case Report.J Clin Diagn Res.2013;7(8):1802-03.

[19]. SanthanakrishnanM, Rangarao S.Mandibular tori:a source of autogenous bone graft.J Indian Soc.Periodontol..2014;18(6):767-71

[20]. Nayak SS,Nayak VS.A Rare case of mandibular exostosis and its review.J Clin Diagn Res.2016;10(1):AJ01-2.

[21]. Al Quran FA,Al-Dwairi ZN.Torus Palatinus and Torus mandibularis in edentulous patients.J Contemp Dent Pract.2006;7(2):112-9. 